\title{
Synthesis and characterization of CdS nanoparticles embedded in a polymethylmethacrylate matrix
}

\author{
Lucia Pedone $^{\mathrm{a}, *}$, Eugenio Caponetti ${ }^{\mathrm{a}}$, Maurizio Leone ${ }^{\mathrm{b}}$, Valeria Militello ${ }^{\mathrm{b}}$, \\ Valentina Pantò ${ }^{a}$, Stefano Polizzi ${ }^{\mathrm{c}}$, Maria Luisa Saladino ${ }^{\mathrm{a}}$ \\ ${ }^{a}$ Università di Palermo, Dipartimento di Chimica Fisica and INSTM-UdR Palermo, Viale delle Scienze, Parco d'Orleans II, I-90128 Palermo, Italy \\ ${ }^{\mathrm{b}}$ Università di Palermo, Dipartimento di Scienze Fisiche ed Astronomiche and INFM-UdR Palermo, Via Archirafi, 36, I-90123 Palermo, Italy \\ ' Università Ca' Foscari di Venezia, Dipartimento di Chimica Fisica, Via Torino, 155/b 30172 Mestre-Venezia, Italy
}

Received 22 March 2004; accepted 20 October 2004

Available online 13 December 2004

\begin{abstract}
CdS nanopowder capped with sodium bis(2-ethylhexyl)sulfosuccinate was synthesized by using water-in-oil microemulsions. The CdS nanoparticles of about $5 \mathrm{~nm}$ obtained were embedded in polymethylmethacrylate matrix by a photocuring process. The transparent yellow solid compound was characterized by optical absorption and emission spectroscopy, high-resolution transmission electron microscopy, and energy-dispersive X-ray spectroscopy. The properties of this compound were compared with those of the nanopowder dispersed in heptane and in methylmethacrylate. The results obtained indicate that the nanoparticles are homogeneously dispersed in the matrix and do not change in size during the embedding process. Even if the surface slightly changes its luminescence properties, as a consequence of the different new chemical environment, the final product seems to be suitable for practical applications.
\end{abstract}

(c) 2004 Elsevier Inc. All rights reserved.

\section{Introduction}

In the last few years, the production of semiconductor nanoparticles was the focus of much research because of their potential applications in optoelectronics [1], photocatalysis [2], solar energy conversion [3], and water pollutant photodegradation [4]. Devices using the properties of nanoparticles are promising due to the possibility of tailoring a number of optical, electrophysical, and magnetic properties by changing nanoparticle size, which can be controlled during synthesis. Although a wide range of synthetic methods are now available, it seems clear that there is still a major problem associated with the reproducible preparation of materials with good mechanical properties for technological applications. A promising way to prepare these kinds of materials uses the interplay between polymer chemistry and quantum dot synthesis [5].

\footnotetext{
* Corresponding author. Fax: +39-091-590015.

E-mail address: lucia.pedone@tin.it (L. Pedone).
}

In contrast to microelectronic circuits, operating via ordered functional elements such as logical gates, polymer nanocomposites use the properties of disordered systems, as the nanoparticles are stochastically dispersed in the system. These properties can be used to manufacture LEDs, photodiodes, solar cells, and sensors and for other technological applications [6].

Two main methods are used to obtain nanoparticles embedded in a matrix [7]: one carries out the nanoparticle synthesis inside the polymeric matrix [8]; the other consists of allowing monomer polymerization after nanoparticle dispersion has occurred [9].

The goal of the present article was to incorporate CdS nanoparticles into a solid matrix where the growth of the particles and their coalescence processes are inhibited. To use the CdS optical properties the matrix must be transparent. CdS nanoparticles, capped with sodium bis(2-ethylhexyl)sulfosuccinate, were embedded in a polymethylmethacrylate (PMMA) matrix by means of a photocuring process, which is photoinduced hardening of a monomeric 
substrate. PMMA, a vinyl polymer obtained by free radical polymerization of the methylmethacrylate monomer (hereafter MMA), is a thermoplastic transparent material used as a shatterproof replacement for glass. Its refractive index is equal to 1.49 , in the range of the typical refractive index of glass (1.3-1.7); whereas its haze $(1-3 \%)$ is higher than that of glass, which ranges from 0 to $0.17 \%$.

$\mathrm{CdS}$ nanoparticles were synthesized in water-in-oil microemulsions, and to stop the growth process an amine was added before extraction. Nanoparticle growth was followed in situ by UV-vis spectroscopy; at the end of the growth process and after the embedding process, the size was confirmed by high-resolution transmission electron microscopy (HRTEM). Before the embedding process, nanoparticle elemental composition was determined using energy-dispersive X-ray (EDX) spectroscopy. Optical properties of the nanoparticles embedded in the polymer matrix were revealed by photoluminescence spectroscopy and compared with those of the nanopowder dispersed in heptane and in MMA.

\section{Experimental}

\subsection{Materials}

Sodium bis(2-ethylhexyl) sulfosuccinate (AOT, Aldrich 98\%), cadmium sulfate (Aldrich, 99\%), tetrabutylammonium hydrogen sulfide (TBAS, Fluka, 99\%), bis(2-ethylhexyl)-amine (BEA, Aldrich, 99\%), and $n$-heptane (Sigma, 99\%) were used as received. Water was bidistilled. Methylmethacrylate $(99.0 \%$, Aldrich) was purified by using a disposable column, to eliminate the polymerization inhibitor.

\subsection{Methods}

UV-vis absorption spectra were obtained in the range 300-600 $\mathrm{nm}$ by using a double-beam Beckman DU-640 spectrophotometer with a resolution of $1.0 \mathrm{~nm}$. Photoluminescence (PL) spectra in the range 400-750 nm were obtained using a Jasco FP-570 spectrofluorometer equipped with a xenon lamp of $150 \mathrm{~W}$ as an excitation light source. All measurements were performed at room temperature under excitation wavelengths of 400 and $425 \mathrm{~nm}$. Each PL spectrum was normalized by dividing the obtained values by the absorption intensity at the corresponding excitation wavelength. Moreover, the emission spectrum of the medium in which the nanoparticles were dissolved, i.e., heptane, MMA, and PMMA, was subtracted.

HRTEM and EDX investigations were carried out with a JEOL 3010 transmission electron microscope with an UHR pole piece (point-to-point resolution of $0.17 \mathrm{~nm}$ ). Samples of AOT-capped CdS nanoparticles for HRTEM observations were obtained by placing a $5-\mu \mathrm{L}$ drop of heptane dilute dispersion of nanoparticles on a carbon copper grid, and then evaporating the solvent at room temperature. The solid
PMMA samples containing CdS nanoparticles were prepared using an ultramicrotome.

\section{Results and discussion}

\subsection{CdS nanoparticle synthesis}

Among the various methods employed to produce sizecontrolled nanoparticles, a promising one is based on the use of water-in-oil (w/o) microemulsions. Nanoparticles of many metal sulfides were synthesized in this way by mixing two w/o microemulsions containing the suitable watersoluble metal salt and sodium sulfide, respectively $[10,11]$. In this procedure nanoparticles need to be separated from the reaction medium. Bare nanoparticles are thermodynamically unstable with respect to growth and they tend to coalesce spontaneously, losing their peculiar properties due to the small dimensions. For this reason nanoparticles are passivated by adding suitable capping agents [12-15], which, bonding to the surface, prevent their coalescence.

$\mathrm{CdS}$ nanoparticles were obtained by adding stoichiometric amounts of solid tetrabutylammonium hydrogen sulfide (TBAS) in a stirred water/AOT $/ n$-heptane microemulsion containing $\mathrm{CdSO}_{4}$. It is well known that nanoparticles size can be controlled by varying different parameters, such as reactant and surfactant concentrations and temperature. In a previous work [16], how the water-to-AOT molar ratio affects nanoparticle size was demonstrated.

The temperature was kept at $25.0 \pm 0.1^{\circ} \mathrm{C}$, the waterto-AOT molar ratio was adjusted to 3 , and the $\mathrm{CdSO}_{4}$ and TBAS concentrations were set at $0.20 \mathrm{~mol} / \mathrm{kg}_{\text {solution. }}$ TBAS readily dissolves and reacts, allowing the formation of $\mathrm{CdS}$ nanoparticles. To stop growth and to separate nanoparticles from the reactant medium, BEA was added to have a BEA/CdS molar ratio of about 4. BEA addition was performed after $1 \mathrm{~h}$ of mixing reactants. After water and $n$-heptane evaporation at $T=40^{\circ} \mathrm{C}$ under a pressure of about 100 mbar, a nanoparticle-surfactant composite was obtained. The composite was dispersed in ethanol in the weight ratio 1 to 15 by gently stirring for $5 \mathrm{~min}$. A certain amount of AOT was solubilized and nanoparticles were separated by centrifugation. To completely remove the surfactant, the process has to be repeated several times. The rinsing process has been stopped at the second cycle to avoid nanoparticle coalescence. The decrease in AOT concentration was inferred by means of UV-vis spectroscopy following the bands that fall in the range $220-250 \mathrm{~nm}$.

\subsection{CdS powder characterization}

AOT-capped CdS nanoparticles dispersed in heptane were characterized by absorption and emission spectroscopy; after solvent evaporation they were characterized by means of HRTEM and EDX techniques. 


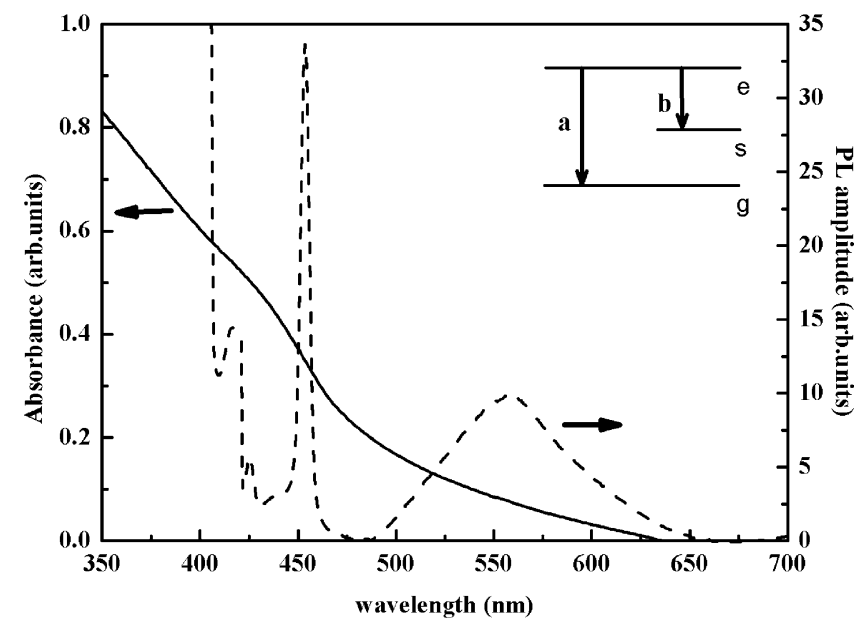

(A)

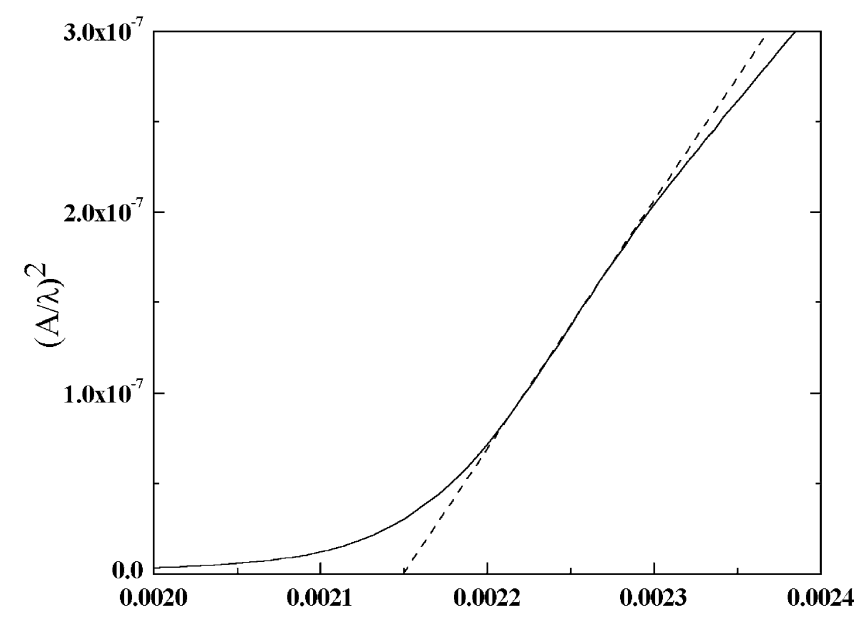

(B)

Fig. 1. (A) Absorption (continuous line) and emission (dashed line) spectra of AOT-capped CdS nanoparticles in heptane. The emission spectrum was obtained using an excitation wavelength of $400 \mathrm{~nm}$. The narrow peak is a band-edge emission from the radiative decay from excited state $\langle e\rangle$ to ground state $\langle g\rangle$. The broad peak is from radiative decay of the carrier captured by the nanocrystal surface trap state $\langle s\rangle$ from the excited state, as shown in the inset. (B) Plot of $(A / \lambda)^{2}$ versus $1 / \lambda$.

The absorption UV-vis spectrum is shown in Fig. 1 as a function of wavelength. The band centered at $450 \mathrm{~nm}$ is due to the CdS first $(1 s-1 s)$ exciton transition [17], i.e., the valence band-conduction band transition. As a consequence of the quantum size effect, the threshold wavelength $\left(\lambda_{\mathrm{s}}\right)$ of this band is a function of nanoparticle size [18-20].

To evaluate the $\lambda_{\mathrm{s}}$ values, UV-vis spectra were analyzed according to the equation

$\left(\frac{A}{\lambda}\right)^{2}=K\left(\frac{1}{\lambda}-\frac{1}{\lambda_{\mathrm{s}}}\right)$

where $A, \lambda$, and $K$ are absorbance, wavelength, and an empirical constant, respectively. $(A / \lambda)^{2}$ is plotted versus $1 / \lambda$ in Fig. 1B; the dashed line represents the linear regression of the rising part of the band. From its intersection with the $x$ axis a $\lambda_{\mathrm{s}}$ value of $464 \pm 9 \mathrm{~nm}$ was achieved.

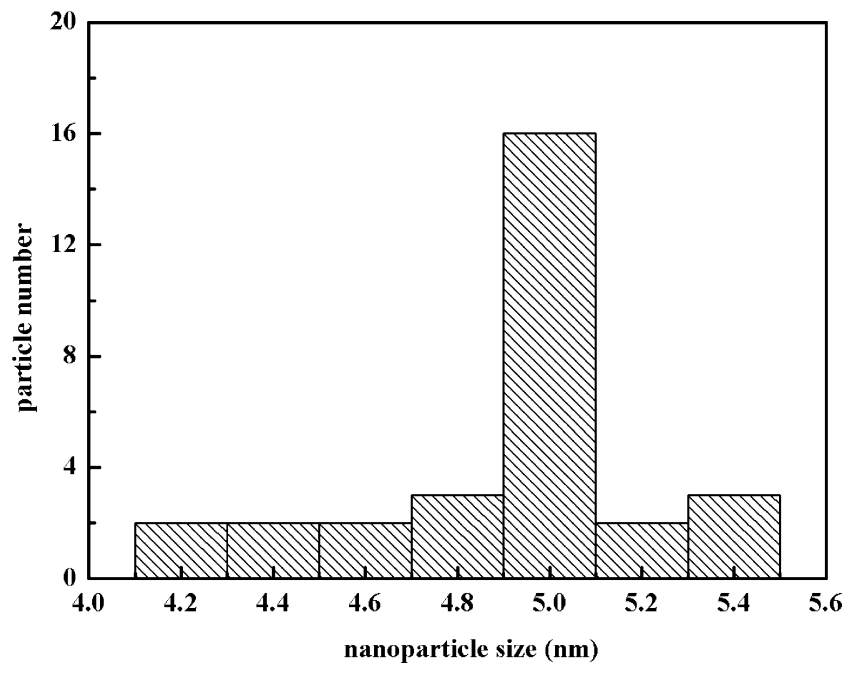

Fig. 2. Particle size distribution of CdS nanoparticles determined by HRTEM. The histogram was obtained from a statistical counting made on several micrographs.

To obtain a relationship between $\lambda_{\mathrm{s}}$ and nanoparticle mean diameters $(d)$, in a previous paper [16] UV-vis spectra and HRTEM measurements were performed with AOTcapped CdS nanoparticles dispersed in $n$-heptane and synthesized using five water/AOT $/ n$-heptane microemulsions having different water/AOT molar ratios. The empirical relationship obtained was

$\lambda_{\mathrm{s}}=\alpha \times d^{\beta}$,

where $\alpha=237 \pm 13$ and $\beta=0.17 \pm 0.02$ [21]. As $d$ is the mean size of the particles, no information on the polydispersity of the sample can be obtained.

With Eq. (2) a nanoparticle size of $5.3 \pm 1.6 \mathrm{~nm}$ was obtained. The error in $d$ was calculated by using the standard error propagation theory considering the uncertainties on $\lambda_{\mathrm{s}}$, $\alpha$, and $\beta$.

In Fig. 1 the photoluminescence activity induced under excitation at $400 \mathrm{~nm}$, i.e., within the optical absorption band centered at $450 \mathrm{~nm}$, is also shown. As can be seen in the inset to Fig. 1, the deexcitation pattern involves two radiative channels:

1. A radiative recombination of an electron-hole pair trapped, which is referred to as "band-edge emission," gives a narrow emission band at about $450 \mathrm{~nm}$ (channel a in the inset).

2. A deexcitation pathway is due to the recombination of the exciton trapped in the defect states localized on the surface of the nanoparticle ("surface luminescence" channel $b$ in the inset) [22-25].

The last deexcitation pathway is responsible for the emission band centered at about $560 \mathrm{~nm}$, whose width is determined by inhomogeneous and homogeneous broadening, due to electron-phonon coupling. Moreover, we cannot exclude the presence of other nonradiative deexcitation pathways. Photoluminescence excitation profile measurements 


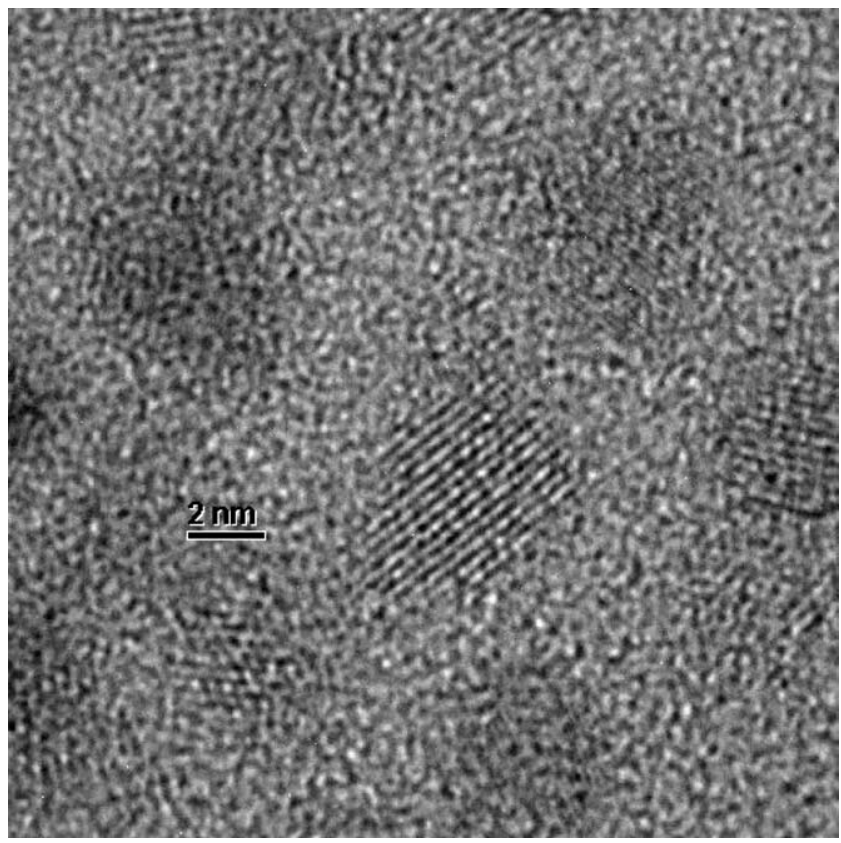

Fig. 3. HRTEM image of AOT-capped CdS nanoparticles.

(data not shown) confirm that the two emission bands have the same excitation band, centered at about $450 \mathrm{~nm}$, in agreement with the observed absorption band.

The histogram in Fig. 2 was obtained from a statistical count made on several HRTEM micrographs. The nanoparticle size distribution centered at $5.0 \mathrm{~nm}$ is characterized by a polydispersity of about $18 \%$. The small discrepancies with data obtained under similar conditions and reported in Ref. [16] are related to differences in the time at which BEA was added. The mean size agrees with the value obtained by means of UV-vis band analysis; this confirms the validity of the above procedure in determining the mean particle size from the CdS absorption band.

From the HRTEM image in Fig. 3 it can be seen that nanoparticles are crystalline with two $d$ spacings of 3.2 and $2.1 \AA$, which are compatible with the CdS cubic structure.

Information on nanoparticle elemental composition before the embedding process was obtained by means of EDX spectroscopy. The pattern in Fig. 4 shows the peaks pertaining to the elements constituting the nanoparticles $(\mathrm{Cd}$ and $\mathrm{S})$ together with peaks linked to the surfactant molecules $(\mathrm{C}, \mathrm{O}, \mathrm{Na})$. The Na peak confirms that, after extraction from the reaction media, AOT molecules are still present.

\subsection{Characterization of CdS nanoparticles dispersed in $M M A$}

The homogeneous dispersion obtained by sonicating a CdS nanopowder-MMA mixture was characterized by means of absorption and emission spectroscopy. PL spectra were obtained using two different excitation wavelengths (400 and $425 \mathrm{~nm}$ ). All spectra are reported in Fig. 5. For comparison, the pure MMA UV-vis spectrum is also re-

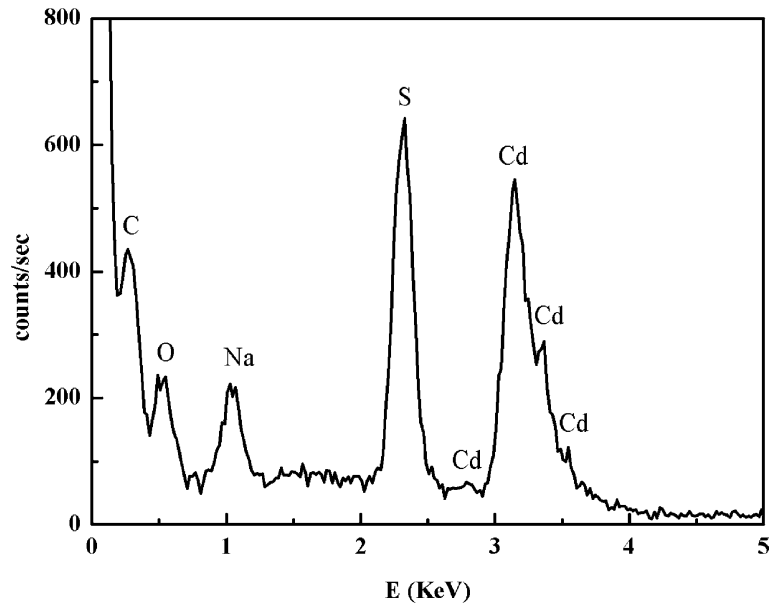

Fig. 4. EDX patterns of CdS nanoparticles dispersed in heptane after addition of BEA and extraction from reaction medium.

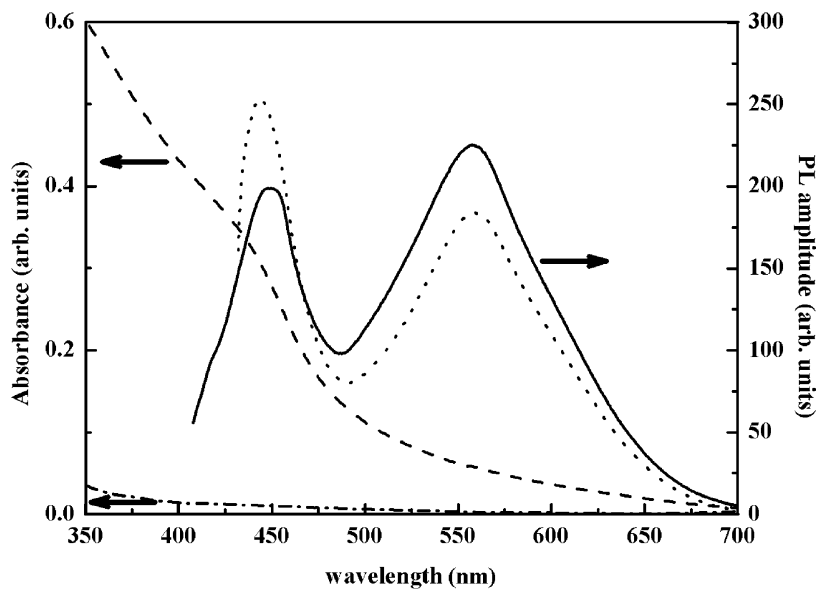

Fig. 5. UV-vis spectra of MMA (dashed-dotted line) and of AOT-capped CdS nanoparticles dispersed in MMA (dashed line) together with the PL spectra (excitation wavelength: $400 \mathrm{~nm}$, continuous line; $425 \mathrm{~nm}$, dotted line).

ported (dashed-dotted line in Fig. 5). No significant differences are present in the CdS absorption band, when compared with the spectrum of nanoparticles dispersed in heptane (solid line in Fig. 1), except for an intensity factor. Analysis of the CdS absorption band, centered at $450 \mathrm{~nm}$, gave an average nanoparticle size of $5.2 \pm 1.6 \mathrm{~nm}$, very close to that obtained for nanoparticles dispersed in heptane; no absorption in the range of interest $(350-700 \mathrm{~nm})$ is observed for pure MMA.

The emission spectrum obtained using the 400-nm excitation wavelength (solid line in Fig. 5), exhibits interesting differences from the spectrum of $\mathrm{CdS}$ nanoparticles dispersed in heptane (dashed line in Fig. 1). The two emission bands at about 450 and $550 \mathrm{~nm}$ are still present, but they both appear broader, with the relative intensity ratio largely modified. It can be deduced that the direct transition is substantially reduced in favor of the alternative deexcitation pathway involving surface defects. In this respect, it must be noted that the PL spectra are corrected by dividing the obtained values 


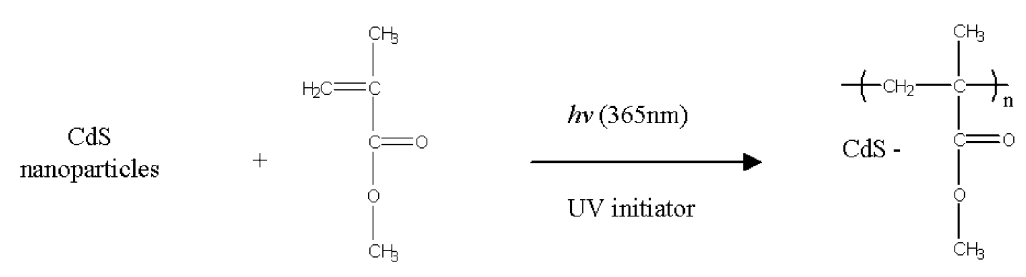

MMA CdS embedded in PMMA

Fig. 6. Scheme of PMMA polymerization in the presence of AOT-capped CdS nanoparticles.

by the absorption intensity at the corresponding excitation wavelength. The broadening of the band profile and the variation of the intensity ratio changes can be ascribed to the modified environment of the particles, when they are dissolved in MMA. Comparing the two PL spectra obtained under excitation at 400 and $425 \mathrm{~nm}$ (continuous and dotted lines in Fig. 5, respectively), we note a significant dependence on excitation wavelength, as evidenced by the inversion of the intensity ratio of the two emissions. As reported in the literature for other systems, this suggests the presence of a conformational distribution of particles having slightly different spectral properties that can be selectively excited at different wavelengths [26-28].

\subsection{Synthesis and characterization of CdS-PMMA nanocomposite}

It has been reported by Katsikas et al. [30] that colloidal $\mathrm{CdS}$ can be used as photoinitiator for polymerization of MMA. With this in mind, a first attempt to carry out polymerization was made by placing the MMA-CdS nanoparticle dispersion in a Rayonet reactor equipped with eight 365-nm lamps of approximatively $35 \mathrm{~W}$ and continuously irradiating for $2 \mathrm{~h}$, until the polymerization occurred. This procedure was not suitable for our purpose, as the long UV irradiation damaged the CdS nanoparticles due to an oxidative loss of sulfur.

To avoid the UV radiation damage of CdS nanoparticles, the photocuring process was successfully performed by using a photoinitiator for PMMA polymerization, as reported by Fang et al. [29]. The process is outlined in Fig. 6. The transparent yellow solid product, composed of a polymeric matrix containing $\mathrm{CdS}$ nanoparticles, was characterized by absorption and emission spectroscopy and by HRTEM.

The typical CdS absorption band, obtained in the UV-vis spectrum, is shown in Fig. 7 (dashed line). The nanoparticle average size estimated from the band threshold wavelength was $5.5 \pm 1.6 \mathrm{~nm}$. The pure PMMA UV-vis spectrum reported in the same figure (dashed-dotted line) shows negligible absorption in the range of interest $(400-700 \mathrm{~nm})$.

The emission spectra (continuous and dotted lines in Fig. 7) seem to be less dependent, with respect to the MMA case, on excitation wavelength. Moreover, both radiative emissions appear to be less efficient when CdS particles are embedded in PMMA, probably due to the presence of larger

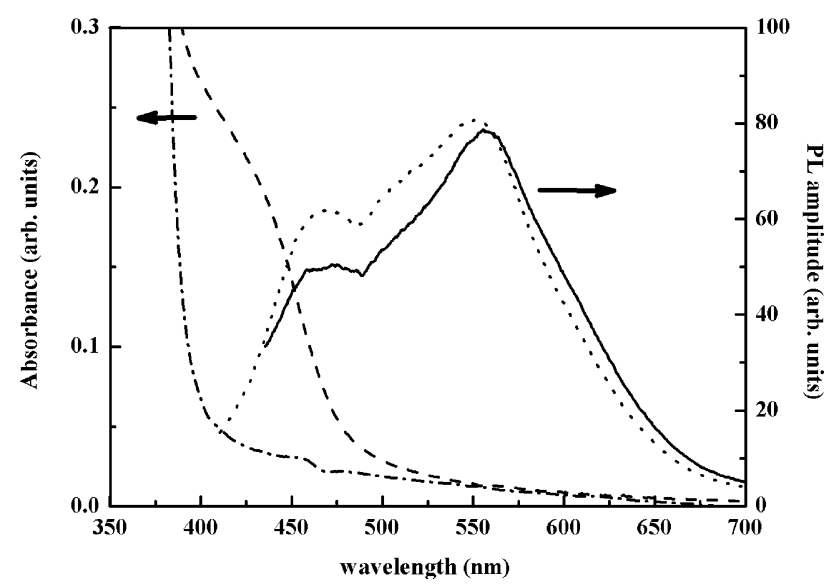

Fig. 7. UV-vis spectra of PMMA (dashed-dotted line) and CdS-PMMA (dashed line) together with the PL spectra of CdS-PMMA (excitation wavelength: $400 \mathrm{~nm}$, continuous line; $425 \mathrm{~nm}$ : dashed line).

nonradiative deexcitation channels. This result validates data relative to the sample dispersed in MMA monomers; i.e., PL activity is sensible to the modification induced on the surface by the environment. In particular, the two emission bands are broad and the radiative channel involving the surface defects appears to be, in percentage, more efficient. Moreover, comparing the ratio of the two radiative channels from PL spectra obtained under excitation at 400 and $425 \mathrm{~nm}$ (continuous and dotted lines in Fig. 7, respectively), it is evident that the dependence on excitation wavelength is less noticeable with respect to the MMA case.

The HRTEM image in Fig. 8 shows that nanoparticles embedded inside the PMMA polymeric matrix are still crystalline and they do not change their shape or their narrow size distribution. The nanoparticle mean size of about $5.0 \mathrm{~nm}$ obtained by HRTEM is consistent with the value estimated by UV-vis band analysis.

\section{Conclusions}

It has been shown that $\mathrm{CdS}$ nanoparticles can be dispersed in MMA monomers and that CdS nanoparticles embedded in a PMMA matrix can be obtained as a transparent yellow solid by a photocuring process, initiated by activating a photoinitiator with $365-\mathrm{nm}$ radiation. Such a material could find interesting optical applications. 


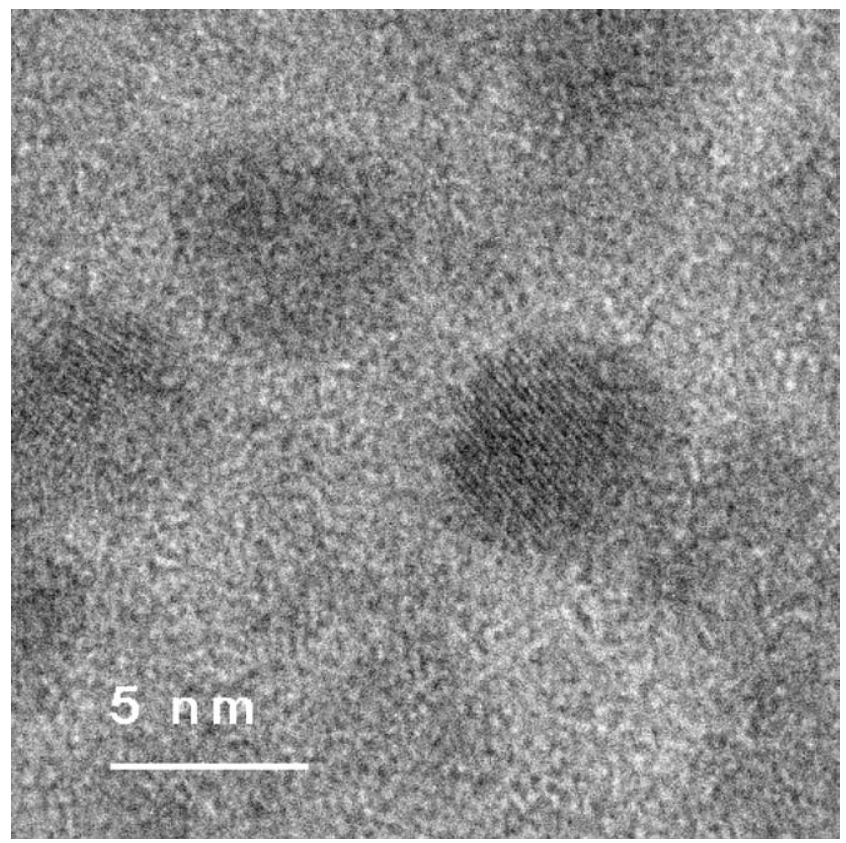

Fig. 8. HRTEM image of CdS nanoparticles embedded in PMMA.

The proposed procedure does not damage the CdS optical properties, is fast, and has the advantage of working at room temperature. Before the extraction process, HRTEM demonstrates that nanoparticles are crystalline and spherical in shape with a narrow size distribution centered at $5.0 \mathrm{~nm}$. The EDX pattern indicates that the CdS nanoparticles, whose growth was stopped with BEA, are capped with sodium bis(2-ethylhexyl) sulfosuccinate (AOT). After the embedding process, nanoparticles maintain their structural properties.

A detailed analysis of the photoluminescence activity of $\mathrm{CdS}$ nanoparticles dispersed in heptane and MMA and embedded in PMMA revealed that the external environment only slightly affects the surface properties. Finally, PMMA turned out to be an interesting polymeric matrix for $\mathrm{CdS}$ quantum dot applications, for its transparency in the spectral region where the optical properties of nanoparticles are important.

\section{Acknowledgments}

The authors are most grateful to Dr. Casinelli (Basel) for preparation of the PMMA ultrathin sections for HRTEM. Financial support from the Consiglio Nazionale delle Ricerche
(Progetto Finalizzato Materiali Speciali per tecnologie avanzate II) and from MURST (PRIN-COFIN 2001 "Sintesi di nanoparticelle assistita da microonde") is gratefully acknowledged.

\section{References}

[1] B.A. Korgel, H.G. Monbouquette, J. Phys. Chem. 100 (1996) 346.

[2] C.K. Grazel, M. Gratzel, J. Am. Chem. Soc. 101 (1979) 7741.

[3] J. Ramsden, M. Gratzel, J. Chem. Soc. Faraday Trans. 180 (1984) 919.

[4] A. Mills, G. Williams, J. Chem. Soc. Faraday Trans. 185 (1989) 503.

[5] J. Lee, V.C. Sundar, J.R. Heine, M.G. Bawendi, K.F. Jensen, Adv. Mater. 1 (2000) 1103.

[6] D.Y. Godovsky, Adv. Polym. Sci. 153 (2000) 163.

[7] G. Kickelbick, Prog. Polym. Sci. 28 (2003) 83.

[8] G. Carotenuto, G. Marletta, L. Nicolais, J. Mater. Sci. Lett. 20 (2001) 663.

[9] J. Fang, L.D. Tung Kevin, L. Stokes, J. He, D. Caruntu, W.L. Zhou, C.J. O'Connor, J. Appl. Phys. 91 (2003) 8816.

[10] D. Kim, M. Miyamoto, M. Nakayama, Phys. Status Solidi C 0 (2003) 1233.

[11] M.P. Pileni, Langmuir 13 (1997) 3266.

[12] G.C. Lisensky, R.L. Penn, C.J. Murphy, A.B. Ellis, Science 248 (1990) 840.

[13] C.R. Cowdery, D.G. Whitten, G.L. McLendon, J. Chem. Phys. 76 (1993) 377.

[14] T. Dannhauser, M. O'Neil, K. Johansonn, D.G. Whitten, G.L. McLendon, J. Phys. Chem. 90 (1986) 6074.

[15] P.V. Kamat, N.M. Dimitrijevic, J. Phys. Chem. 93 (1989) 42.

[16] E. Caponetti, L. Pedone, D. Chillura Martino, V. Pantò, V. Turco Liveri, Mater. Sci. Eng. C 23 (2003) 531.

[17] J.H. Fendler, Chem. Rev. 87 (1987) 877.

[18] Y. Wang, N. Herron, J. Phys. Chem. 95 (1991) 525.

[19] L.E. Brus, J. Chem. Phys. 80 (1984) 4403.

[20] D.Y. Godovski, Adv. Polym. Sci. 119 (1995) 110.

[21] E. Caponetti, unpublished data.

[22] Y. Lin, J. Zhang, E.H. Sargent, E. Kumacheva, Appl. Phys. Lett. 81 (2002) 3134

[23] S.F. Wuister, A. Meijerink, J. Lumin. 102/103 (2003) 338.

[24] T. Orii, S. Kaito, K. Matsuishi, S. Onari, T. Arai, J. Phys. Condens. Matter. 14 (2002) 9743.

[25] L. Spanhel, M. Haase, H. Weller, A. Henglein, J. Am. Chem. Soc. 109 (1987) 5649 .

[26] J.R. Lakowicz, I. Gryczynski, Z. Gryczynski, C.J. Murphy, J. Phys. Chem. B 103 (1999) 7613

[27] M. Leone, S. Agnello, R. Boscaino, M. Cannas, F.M. Gelardi, Phys. Rev. B 60 (1999) 11475

[28] S. Agnello, R. Boscaino, M. Cannas, F.M. Gelardi, M. Leone, B. Boizot, Phys. Rev. B 67 (2003) 33202.

[29] L. Katsikas, J.S. Velickovic, H. Weller, I.G. Popovic, J. Therm. Anal. 49 (1997) 317.

[30] J. Fang, L.D. Tung Kevin, L. Stokes, J. He, D. Caruntu, W.L. Zhou, C.J. O'Connor, J. Appl. Phys. 91 (2003) 8816. 